\title{
The preservation of amplitude information in burst phenomena in physiological recordings
}

\author{
F. JAMES DOYLE and RICHARD ALLON \\ Toronto General Hospital, Toronto, Ontario, Canada MSG $1 L 7$ \\ and University of Toronto, Toronto, Ontario, Canada M5S IAI
}

\begin{abstract}
This paper describes an inexpensive and simple electronic circuit that rectifies and smooths high-frequency burst data and holds the peak value of the burst for predetermined time intervals.
\end{abstract}

There are electrical analogs of physiological events that occur in relatively high-frequency bursts. Examples include EMG records associated with Parkinsonism (Lance, Schwab, \& Peterson, 1963) and other dysfunctions in which tremors occur, EMG associated with repetitive speech and acoustic output (Daniel \& Guitar, 1978), Korotkoff sounds, and others. In such cases, characteristics of the burst envelope such as onset, duration, number of peaks, and peak amplitude are often of more interest than the constituent electrical content. Sometimes limitations of equipment do not permit the accurate reproduction of these signals. For example, the upper frequency limit of $75.100 \mathrm{~Hz}$ of some penwriters causes distortion of a signal such as a Korotkoff sound, which has harmonic frequency content of $60.300 \mathrm{~Hz}$ (McCutcheon, Baker, \& Wiederhielm, 1969). This report presents a simple electronic circuit that conditions bursttype data so that the peak amplitude of the envelope may be held either for the duration of the burst or until a predetermined period has elapsed relative to the onset of the burst. Such a circuit allows data that normally fall outside the frequency range of a penwriter to be plotted. This circuit is also useful for microcomputers with relatively slow analog-to-digital (AD) conversion or poor interrupt handling capability to obtain the peak of the envelope while polling and manipulating several other parameters at once. Finally, the circuit facilitates manual determination of an envelope peak.

\section{GENERAL OPERATING CHARACTERISTICS}

Figure 1 presents three examples of the type of data with which the circuit has been used; it also illustrates the three stages of conditioning achieved by the circuit. Figure la provides examples of an EMG burst, monitored

The authors gratefully acknowledge the financial assistance of the Psychiatric Consultants, Toronto General Hospital, in subsidizing personnel costs. Requests for reprints should be sent to F. James Doyle, Research Division, Department of Psychiatry, Toronto General Hospital, 101 College Street, Toronto, Ontario, Canada M5G 1L7. F. J. Doyle is with the Department of Psychiatry at the University of Toronto; R. Allon is with the Departments of Psychiatry and Behavioral Science. using surface cup electrodes on the biceps. Figure $1 \mathrm{~b}$ shows Korotkoff sounds monitored with a Biocom 1010 crystal pulse pick-up transducer over the brachial artery during a blood pressure determination. Figure $1 \mathrm{c}$ depicts an episode of repetitive speech usage as monitored and recorded using a crystal microphone. In order to plot the raw data for Figure 1c, the original data were recorded on tape and then slowed by a factor of eight before outputting to the penwriter. This manipulation was done for illustrative purposes only. Normally, only the peak waveform is monitored on the penwriter. The various stages of conditioning achieved by the circuit are illustrated in Figure 1. The raw data (Channel 1, bottom channel) is rectified (Channel 2) and smoothed (Channel 3); when the smoothed waveform crosses a threshold, a timer begins timing, and the circuit remains in the peak detection mode (Channel 4) until the timer period is finished. A threshold level that is a valuable tool for marking the onset of the burst is doubly useful for rejecting noise.

\section{CIRCUIT OPERATION}

The circuit schematic is shown in Figure 2. The principal components used are two internally compensated quad operational amplifiers (MC3404P), a field effect transistor (FET) input operational amplifier (LF355H), and a monostable multivibrator (MC14528BCP). Substitutions for any of these components are readily available. The functions for the various stages are as follows. Amplifier A1 and its associated circuitry form a noninverting ac coupled amplifier with a dc biasing network and a gain factor of two. This stage blocks any dc bias present on the input signal and permits adjustment of the bias level for the next stages in the circuit. If for any reason the gain factor of two is to be reduced to unity gain, an attenuator consisting of two 10-kohm resistors connected in series between the output of $\mathrm{Al}$ and ground will do this, providing that the output to the next stage is taken from the connection between the two resistors. Later in the circuit, the envelope amplitude is reduced by smoothing, and the gain may have to be increased. The stage incorporating Amplifiers 


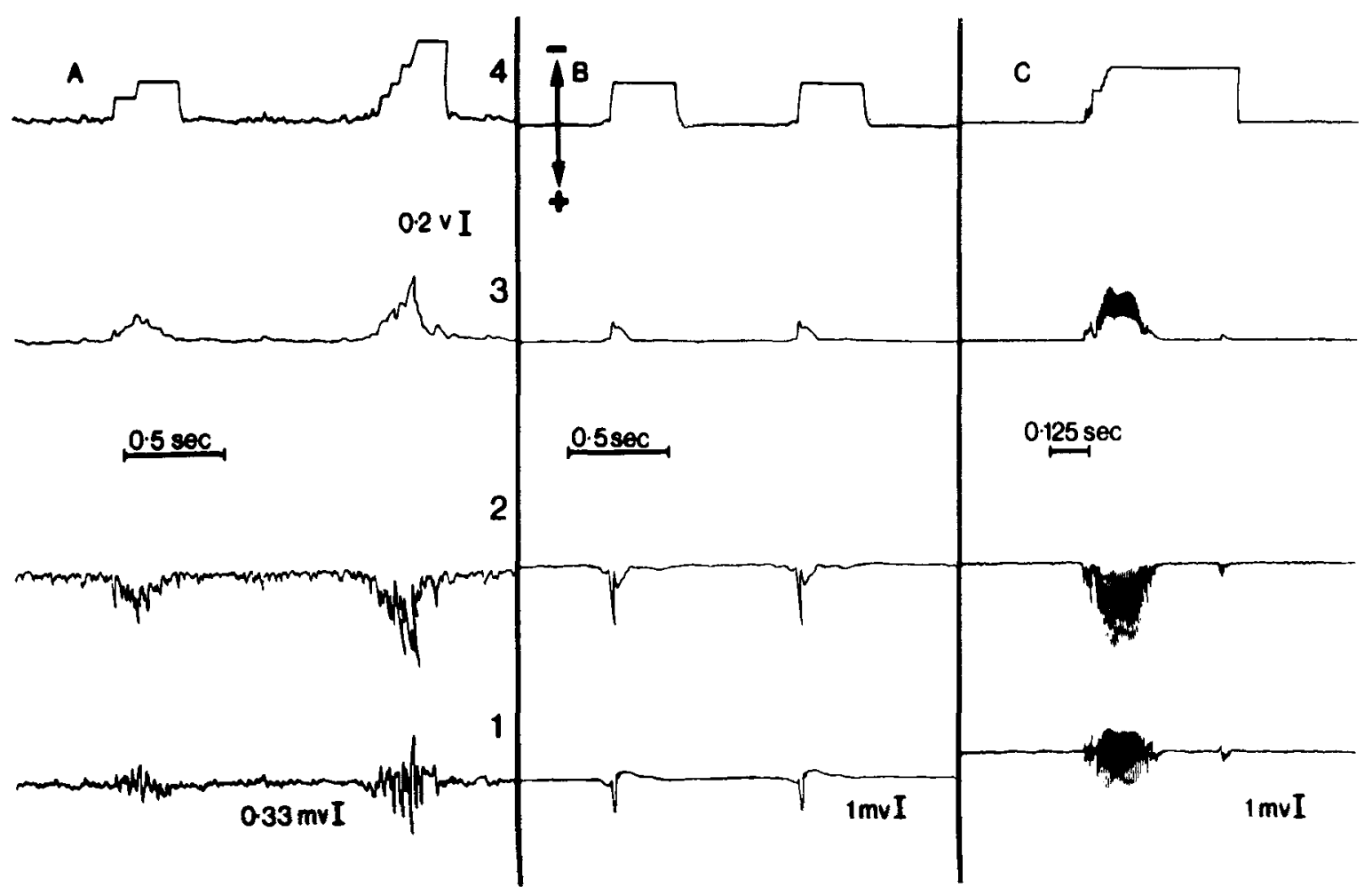

Figure 1. Examples of EMG, Korotkoff sounds, and repetitive speech and the levels of signal conditioning.

A2 and A3 constitutes a full-wave dc rectifier (Giles, 1967) or absolute amplifier. Full-wave rectification is useful for smoothing network operation because more data signal is applied to the integrator so that a smoother signal of higher amplitude is achieved. The biasing adjustment of Amplifier $\mathrm{Al}$ is set so that when a signal of equal amplitude in both polarities is presented to the input of $\mathrm{Al}$, the output of Amplifier A3 must be equal in amplitude for both polarities.

The output from Amplifier A3 connects to the smoothing integrator circuit consisting of Resistor R1 and Capacitor $\mathrm{Cl}$. Table 1 presents some typical values for these components when used with various physiological parameters. A multiple-position switch to in troduce various degrees of smoothing (by changing values at will for $\mathrm{C} 1$ ) would permit the circuit to be used for various parameters. Amplifier A4 is a buffer amplifier to maintain the impedance characteristics of the smoothing $\mathrm{RC}$ network and to reproduce the change in potential across C1. Amplifier A5 on the far right of Figure 2 is an inverting amplifier that permits adjustment of the peak waveform as presented at the output. This stage inverts the polarity of the smoothed waveform for the peak detector and provides an adjustment of the amplitude of the output signal.

The circuit block in the lower right corner of Figure 2 consisting of Amplifier A6. FET F1, and Transistor T1 is a comparator network with a fixed reference level. When the smoothed waveform envelope is presented to this circuit and is of st 'ficient amplitude to cross a threshold set by FET F1, the comparator A6 changes state. This state change starts the timing operation for the peak detection sequence. Transistor $\mathrm{T} 1$ is a buffer stage to convert the bipolar output of $\mathrm{A} 6$ to a unipolar level that can trigger the monostable timer circuit. The threshold level of about $.1 \mathrm{~V}$ can be made variable by substituting a $1-\mathrm{kohm}$ potentiometer in place of the 47-ohm resistor at the lower right corner of Figure 2. Examination of Channel 4 of Figure 1 shows that the peakdetection mode is not entered until this level is crossed.

The circuit in the lower left block of Figure 2 consists of the peak-detection circuit and timer. Amplifiers A7 and $A 8$ form a peak-detection circuit. Amplifier A8 is a FET operational amplifier with a characteristically high input impedance. Any degradation of the held peak waveform is a function of the leakage currents at the input of A8. So, if a high-quality capacitor is used for the 1-microF storage capacitor, the peak value can be held for very long periods, hours if necessary. The acquisition time of the circuit as presented in Figure 2 is about $.7 \mathrm{msec}$. In order not to latch on transient noise, the peak-detection acquisition period was deliberately slowed down by introducing the 10-kohm resistor between Amplifiers A7 and A8. When a signal of insufficient amplitude to cross the threshold reference level is applied at the circuit input, the 1-microF storage capacitor remains shorted to ground and any previous peak value is cleared. In this mode the peak detector appears as an ordinary unity gain amplifier. When a signal of sufficient amplitude arrives, FET F2 open circuits and the peak value is held. FET F2 is controlled by the timing monostable MC14528BCP. With the 


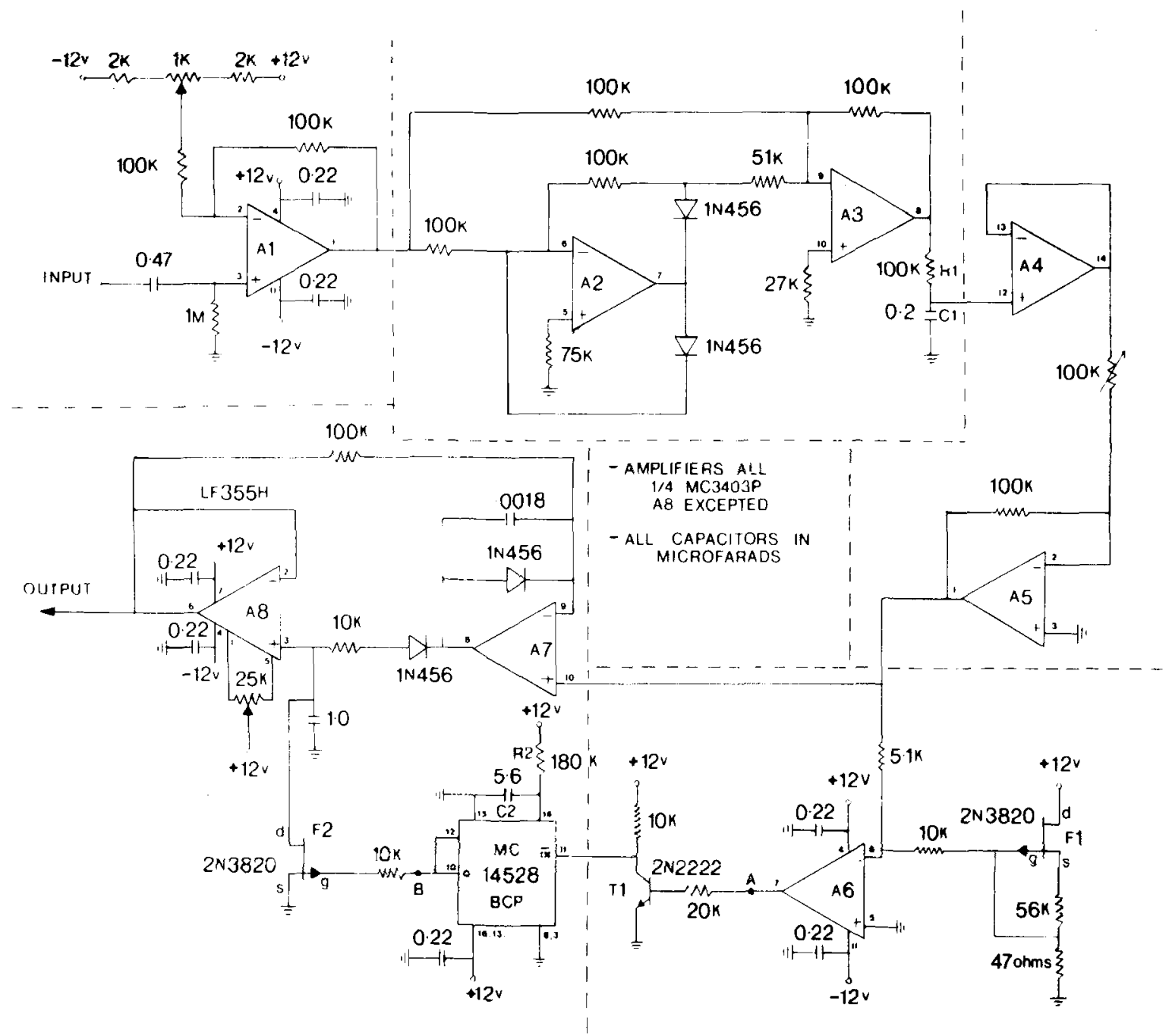

Figure 2. Circuit schematic.

Table 1

Smoothing Time Constant Values for R1 $=100 \mathrm{kohm}$

\begin{tabular}{cclc}
$\begin{array}{c}\text { Spectrum } \\
(\mathrm{Hz})\end{array}$ & $\begin{array}{c}\mathrm{C} 1 \\
\text { (MicroF) }\end{array}$ & \multicolumn{1}{c}{ Use } & $\begin{array}{c}\text { Tau } \\
\text { (Sec) }\end{array}$ \\
\hline $15-75$ & .2 & EMG & .02 \\
$15-75$ & 5 & EMG & .05 \\
$60-300$ & .1 & K. Sounds & .01 \\
$100-5000$ & .05 & Speech & .005 \\
\hline
\end{tabular}

values for $\mathrm{R} 2$ and $\mathrm{C} 2$ as shown in Figure 2, the timing period is about $300 \mathrm{msec}$. Presented in Table 2 are some timing values for a few standard resistive and capacitive values. In the event that the peak value is to be held only during the period of the burst, this may be accomplished by removing the circuit between the points marked $A$ and $B$ and connecting $A$ to $B$. When this is done, the search for the peak value begins when the reference level is traversed and as soon as the signal falls below the reference level, the stored peak is cleared.

The circuit, as shown in Figure 2, is designed to produce a negative-going signal at the output in order to conform with the medical convention that negativity causes an upward deffection on a penwriter. Some older types of $\mathrm{AD}$ convertors and penwriters expect a unipolar positive-going signal. Because there is one free operational amplifier in one of the integrated circuit packages, a circuit identical to that which surrounds Amplifier A5 in

Table 2

Monostable Capacitance and Time Values for R2 $=100 \mathrm{kohm}$

\begin{tabular}{cccc}
$\begin{array}{c}\mathrm{C} 2 \\
\text { (MicroF) }\end{array}$ & $\begin{array}{c}\mathrm{T} \\
(\mathrm{Sec})\end{array}$ & $\begin{array}{c}\mathrm{C} 2 \\
(\mathrm{MicroF})\end{array}$ & $\begin{array}{c}\mathrm{T} \\
(\mathrm{Sec})\end{array}$ \\
\hline .01 & .0006 & .05 & .0024 \\
.1 & .0044 & .5 & .0172 \\
1.0 & .031 & 5.0 & .12 \\
10.0 & .22 & 50.0 & .86 \\
100.0 & 1.55 & 500.0 & 6.1 \\
\hline
\end{tabular}


Figure 2 and connected at the output of $A 8$ will reverse the polarity. We use a dual $\pm 12-\mathrm{V}$ dc power supply to operate this circuit, but performance is satisfactory using dual supplies in a range of about $\pm 8- \pm 15 \mathrm{~V}$ dc. It is good practice to decouple each power supply input for every integrated circuit to ground using a .2-microF capacitor, as shown in Figure 2. If the electrical environment in which the circuit is to be used is particularly noisy, then decoupling the power supply inputs of the mounting board to ground using a large capacitive value such as 100 microF can also be useful.

\section{CONCLUSION}

The use of this relatively inexpensive and simple circuit permits the analysis of high-frequency physiological data bursts with more accuracy and ease than would the analysis of such data in unconditioned form.

\section{REFERENCES}

Daniel, W., \& Guitar, B. EMG feedback and recovery of facial and speech gestures following neural anastomosis. Journal of Speech and Hearing Disorders, 1978, 43, 9-20.

GILES, J. N. Applications. In J. N. Giles (Ed.), Linear integrated circuits applications handbook. Mountain View, Calif: Fairchild Semiconductor, 1967.

Lance, J. W., Schwab, R. S., \& Peterson, E. A. Action tremor and the cogwheel phenomenon in Parkinson's disease. Brain, $1963,86,95-110$.

McCutcheon, E. P., Baker, D. W., \& Wiederhielm, C. A. Frequency spectrum changes of Korotkoff sounds with muffling. Medical Research Engineering, 1969, 8, 30-33.

(Received for publication September 7, 1979; revision accepted January 22,1980 .) 